\title{
Fundamentos de las redes neuronales artificiales: hardware y software
}

\author{
Bonifacio Martín del Brío \\ Dpto. Ingeniería Eléctrica y Electrónica \\ Escuela Universitaria de Ingeniería Técnica Industrial \\ Universidad de Zaragoza \\ 50009 Zaragoza
}

\section{Carlos Serrano Cinca}

Dpto. de Contabilidad y Finanzas

Facultad de C. Económicas y Empresariales

Universidad de Zaragoza

50005 Zaragoza

\subsection{Resumen}

Las redes neuronales artificiales tratan de modelizar esquemáticamente la estructura hardware del cerebro, para reproducir sus características computacionales. Estos sistemas de procesamiento de información, paralelos, distribuidos y adaptativos, a partir de datos del entorno y empleando algoritmos numéricos, son capaces de aprender de la experiencia. En este artículo realizamos una introducción a los sistemas neuronales artificiales. En primer lugar, exponemos sus aspectos esenciales, en cuanto a la estructura de la neurona, arquitectura de la red y aprendizaje. A continuación describimos con brevedad algunos de los modelos más conocidos. Por último, comentamos las diferentes maneras de realizar un sistema neuronal, y sus aplicaciones a la resolución de problemas prácticos.

Palabras clave: Redes neuronales artificiales. Procesamiento de la Información. Neurocomputadores.

\subsection{Abstract}

Neural networks schematically model the hardware structure of our brain to reproduce its computational abilities. These parallel, distributed and adaptative processing systems are able to learn from experience, working on environmental data and by using numerical algorithms. This paper is an introduction to artificial neural networks. Firstly, basic features of the neuron model, network architectu-

Scire. $1: 1$ (en.-jun. 1995). 
re and learning algorithms are presented. Secondly, the best known models of neural networks are described. Lastly, the different procedures for developing an artificial neural system are disscussed, together with their possible applications to real world problems.

Keywords: Artificial Neural Networks. Information Processing. Neural computers.

\section{Introducción}

En la actualidad, muchos de los esfuerzos más dinámicos dentro de las actividades de $\mathrm{I}+\mathrm{D}$ involucran el estudio de materias que hasta hace poco podían considerarse minoritarias, como el caos, los sistemas difusos y las redes neuronales. En el presente trabajo, trataremos sobre la última de las citadas, las redes neuronales o sistemas neuronales artificiales (SNA).

El estudio de los SNA resulta interesante por dos razones principales. Por una parte, para muchos investigadores en neurociencias y psicología constituyen una modelización del cerebro que les permite profundizar en su estudio. Por otra parte, estos modelos resultan importantes desde un punto de vista tecnológico. Pese al gran desarrollo de la electrónica y las ciencias de la computación, ningún sistema creado por el hombre ha sido todavía capaz de llevar a cabo tareas tan aparentemente simples como reconocer una mosca y atraparla al vuelo, tareas que un sistema sencillo como el cerebro de la rana es capaz de llevar a cabo con eficacia. Parece ser que los problemas más sencillos para los animales (visión, reconocimiento de patrones, coordinación, etc.) son precisamente los más difíciles de resolver con las técnicas convencionales de cómputo, basadas en ordenadores que ejecutan secuencialmente complejos algoritmos. De este hecho puede deducirse que la emulación de la estructura de los sistemas de procesamiento biológicos puede resultar interesante para alcanzar similares prestaciones en aquellos problemas que más eficazmente resuelven.

Con las premisas anteriores, los SNA toman como base la estructura computacional del sistema nervioso con la intención de construir sistemas de procesamiento de la información paralelos, distribuidos y adaptativos, que puedan presentar un cierto comportamiento inteligente, similar al que manifiestan los organismos biológicos.

Los SNA constituyen en la actualidad un campo muy activo, en el que confluyen investigadores procedentes de muy diferentes áreas, como la electrónica, física, matemáticas, ingeniería, biología o psicología. Esta gran actividad se manifiesta de varias maneras. En primer lugar, continuamente se introducen nuevos modelos y desarrollos teóricos. Por otra parte, las grandes compañías del sector de la electrónica (Intel, Philips, Siemens, etc.) construyen circuitos electróni-

Scire. 1 : 1 (en.-jun. 1995). 
cos de estructura neuronal que comienzan a comercializar. Por último, pero no por ello menos importante, numerosas empresas aplican ya los modelos neuronales de forma rutinaria a la resolución de importantes problemas técnicos, como una metodología más de entre las muchas disponibles.

En este trabajo realizaremos un repaso general al origen, bases, aplicaciones y realizaciones hardware y software de los sistemas neuronales artificiales. La extensión del artículo no permite profundizar excesivamente en ningún modelo en concreto, por lo que se facilitará abundante bibliografía para el lector interesado.

\section{El $A B C$ de la inteligencia}

Empieza a extenderse la opinión de que las redes neuronales y los sistemas difusos van a desempeñar un papel cada vez más importante, tanto en la comprensión de la cognición como en la construcción de máquinas que emulen la capacidad humana de toma de decisiones en entornos imprecisos y con ruido (Kosko, 1992). Es este uno de los objetivos que se planteó la Inteligencia Artificial (IA) desde su creación hacia la mitad de los años cincuenta, atrayendo la atención de muchas personas y un gran apoyo financiero. La IA ha obtenido desde entonces importantes logros, quizás el más destacable sea el de los sistemas expertos, programas en los que se codifica el conocimiento de especialistas en una cierta materia (diagnóstico de enfermedades, cálculo, diseño, etc.) en forma de reglas de decisión.

La IA progresó rápidamente hasta 1975, pero desde entonces su desarrollo se ha visto frenado en cierta medida (ver, por ejemplo: Marijuán, 1992; Marks, 1993; Gallant, 1993) (1). En la actualidad muchos investigadores piensan que la IA tradicional no ha cumplido totalmente con las expectativas que en un princi-

\begin{tabular}{||l||l||}
\hline \multicolumn{1}{|c||}{ Inteligencia artificial } & Redes neuronales \\
\hline \hline Enfoque descendente & Enfoque ascendente \\
\hline Basado en la psicología & Basado en la biología \\
\hline Qué hace el cerebro & Cómo lo hace el cerebro \\
\hline Reglas Si/Entonces & Generalización a partir de ejemplos \\
\hline Sistemas programados & Sistemas entrenados \\
\hline Lógica, conceptos, reglas & Reconocimiento de patrones, gestalt \\
\hline $\begin{array}{l}\text { Arquitectura von Neumann. Separa-ción } \\
\text { hardware/software }\end{array}$ & $\begin{array}{l}\text { Arquitecturas paralelas, distribuidas y } \\
\text { adaptativas }\end{array}$ \\
\hline
\end{tabular}

Tabla 1. Características de la IA convencional y de los SNA.

Scire. $1: 1$ (en.-jun. 1995). 
pio despertó. A modo de ejemplo, no ha resuelto de manera plenamente satisfactoria problemas de mundo real en campos como visión artificial o reconocimiento del habla, ni tampoco su contribución ha resultado definitiva para lograr una mejor comprensión de los fenómenos cognitivos. La IA se ha centrado tradicionalmente en la manipulación de símbolos y la lógica de primer orden, lo que ha limitado su capacidad de tratar con problemas de mundo real, en los que la incerteza e imprecisión no son despreciables.

Junto a esta aparente pérdida de dinamismo de la IA convencional, hay que señalar el resurgimiento a mediados de la década de los ochenta de las redes neuronales, en paralelo al asentamiento de otras formas de computación alternativas a la clásica estructura von Neumann, como los algoritmos genéticos, la computación evolutiva o los sistemas difusos.

La opinión que empieza a extenderse (Marijuán, 1992; Conrad, 1992) es que las propuestas de la IA no bastan por sí solas para alcanzar una visión general de la naturaleza de la inteligencia y de las ciencias cognitivas. Por su naturaleza funcionalista, la IA se ha despreocupado tanto de los aspectos biológicos de la inteligencia como de la importancia de la relación entre estructura y función. Como contrapartida, disciplinas como los SNA o la vida artificial se desplazan hacia objetivos más próximos a la biología (Marijuán, 1992), por lo que parecen abrir las puertas a una más amplia concepción de la computación.

En definitiva, hoy en día se puede señalar la coexistencia de dos corrientes importantes, no excluyentes, dentro de la búsqueda de la inteligencia. Por una parte, la IA convencional basada en algoritmos que se ejecutan sobre ordenadores von Neumann (2), que representa un enfoque descendente. Por otra, las redes neuronales y otras técnicas de tipo ascendente, que se incluyen en lo que se viene a llamar inteligencia computacional (Marks, 1993) o computación emergente, que se basan en la confección de sistemas compuestos por conjuntos de procesadores elementales que operan en paralelo, imitando las construcciones desarrolladas por la naturaleza. Por lo tanto, se puede hablar en la actualidad del ABC de la inteligencia, haciendo referencia a sus tres aspectos: Artificial, Biológica y Computacional (Marks, 1993).

De lo expuesto hasta el momento, y desde un punto de vista meramente tecnológico, como técnicas de resolución de problemas prácticos, la pregunta que surge es cuál de los dos enfoques resulta más adecuado. Pensamos que ambos son complementarios, como sucede en nuestro propio sistema nervioso (cuyas capacidades pretendemos reproducir), donde coexisten. Una parte de él, la relacionada con los aspectos sensoriales y motores, se sitúa muy próxima a los SNA: nuestro cerebro no posee un algoritmo de reconocimiento del habla o que interprete caracteres manuscritos, sino que de su propia estructura hardware (las redes de

Scire. 1 : 1 (en.-jun. 1995). 
neuronas) emerge el procesamiento que lleva a cabo. El razonamiento a alto nivel que realizamos de manera consciente es, sin embargo, el feudo de la IA convencional. Esta complementariedad se ve plasmada con el reciente desarrollo de los denominados sistemas expertos conexionistas, que combinan redes neuronales y sistemas expertos (Gallant, 1993).

\section{Sistemas paralelos, distribuidos y adaptativos}

\subsection{Cerebro y computador}

Pese a la creencia común, el cerebro y el computador son muy diferentes, como puede apreciarse de la observación de la Tabla 2. Un computador convencional es, a grandes rasgos, una máquina tipo von Neumann, construida alrededor de una única CPU o procesador, que ejecuta secuencialmente un programa almacenado en memoria. Sin embargo, el cerebro no está compuesto por un único procesador, sino por miles de millones de ellos, las neuronas. Estos procesadores resultan ser mucho más simples, lentos y, además, menos fiables que una CPU. Pese a ello, existen problemas difícilmente abordables mediante un computador convencional, pero que el cerebro resuelve con gran eficiencia, tareas como el reconocimiento del habla, la visión de objetos inmersos en ambiente natural o la respuesta a estímulos del entorno.

La idea que subyace en los SNA es que, para resolver el tipo de problemas que los cerebros resuelven con eficiencia, puede resultar conveniente construir sistemas que copien (esquemáticamente) la estructura de la red neuronal biológica para alcanzar una funcionalidad similar. En el siguiente punto describiremos algunas de sus características más destacables.

\subsection{Las redes neuronales como sistemas paralelos, distribuidos y adaptativos}

Tres conceptos importantes en los sistemas nerviosos, y que se desea reproducir en los artificiales, son: cálculo paralelo, memoria distribuida y adaptabilidad. Así, las redes neuronales se describen como sistemas paralelos, distribuidos $\mathrm{y}$ adaptativos (Rumelhart, 1986).

El procesamiento paralelo resulta esencial, como puede apreciarse del siguiente ejemplo. Un ordenador tipo PC emplearía varios minutos en realizar una sencilla tarea de tratamiento en bajo nivel de una imagen compuesta por $256 \times 256$ pixeles. Por ejemplo, un sistema basado en 16 DSP (3) operando en paralelo emplearía únicamente unos 20 milisegundos. Sin embargo, éste es el mismo tiempo que el cerebro tarda en procesar una imagen compuesta por millones de pixeles (provenientes de los conos y bastones de la retina), extraer sus rasgos relevantes, analizarla e interpretarla. Ningún sistema creado por el hombre es

Scire. $1: 1$ (en.-jun. 1995). 


\begin{tabular}{||l||l|l||}
\cline { 2 - 3 } \multicolumn{1}{c|}{} & Cerebro & Computador \\
\hline Velocidad de proceso & $10^{-3}$ segundos & $10^{-9}$ segundos \\
\hline Estilo de procesamiento & paralelo & secuencial \\
\hline Número de procesadores & $10^{11}-10^{14}$ & pocos \\
\hline Conexiones & 10.000 por procesador & pocas \\
\hline Almacenamiento del conocimiento & distribuido & direcciones fijas \\
\hline Tolerancia a fallos & amplia & nula \\
\hline Tipo de control del proceso & democrático & autocrático \\
\hline
\end{tabular}

Tabla 2. Cerebro frente a computador convencional.

capaz de realizar esta tarea en un tiempo similar. Es fácil deducir que la clave reside en los miles de millones de neuronas que intervienen en el proceso de visión operando en paralelo (solamente en la retina intervienen millones de ellas).

Otro concepto importante es el de memoria distribuida. En un computador la información se halla alojada en casillas de memoria de posiciones bien definidas, de manera que si borramos el contenido de una casilla perdemos el dato contenido en ella. Sin embargo, en los sistemas neuronales la información se encuentra distribuida por las sinapsis de la red, de modo que si una sinapsis se ve dañada, no perdemos más que una parte muy pequeña de la información. Los sistemas neuronales biológicos son redundantes, muchas neuronas y sinapsis realizan un papel similar, de modo que el sistema resulta tolerante a fallos (por ejemplo, cada día mueren miles de neuronas en nuestro cerebro, y sin embargo tienen que pasar muchos años para que se resientan nuestras capacidades).

El último concepto fundamental al que haremos referencia es el de adaptabilidad. Los sistemas neuronales son capaces de adaptarse al entorno modificando sus sinapsis, aprendiendo de la experiencia. A diferencia de los sistemas programados, como los computadores, los neuronales aprenden y generalizan a partir de ejemplos.

\section{Estructura de un sistema neuronal artificial.}

En esta sección describiremos la estructura de un sistema neuronal artificial. La pieza básica de las redes biológicas es la neurona. Las neuronas se organizan en capas, varias de ellas constituyen una red neuronal, que puede estar compuesta por millones de neuronas, conformando un subsistema con funcionalidad propia. El conjunto de subsistemas forma el sistema global (sistema nervioso). 
Desde el punto de vista de la confección de SNA orientados a la resolución de problemas prácticos, se establece una estructura jerárquica similar. El ladrillo básico es la neurona artificial, que se organiza en capas; varias capas constituyen una red neuronal, y una o más redes neuronales, junto con los interfaces de entrada/salida y otros módulos adicionales constituyen el sistema global.

Un sistema neuronal (o un sistema conexionista, en general) se compone de los siguientes elementos: conjunto de neuronas, patrón de conexiones (arquitectura), dinámicas de recuerdo y de aprendizaje, y entorno. Estos son los puntos que trataremos en adelante.

Para el lector interesado en profundizar en el tema, citaremos una serie de referencias básicas. Una introducción clásica a los SNA es el artículo de Lippmann (1987), que ha sido recientemente puesta al día en Hush (1993). Dos
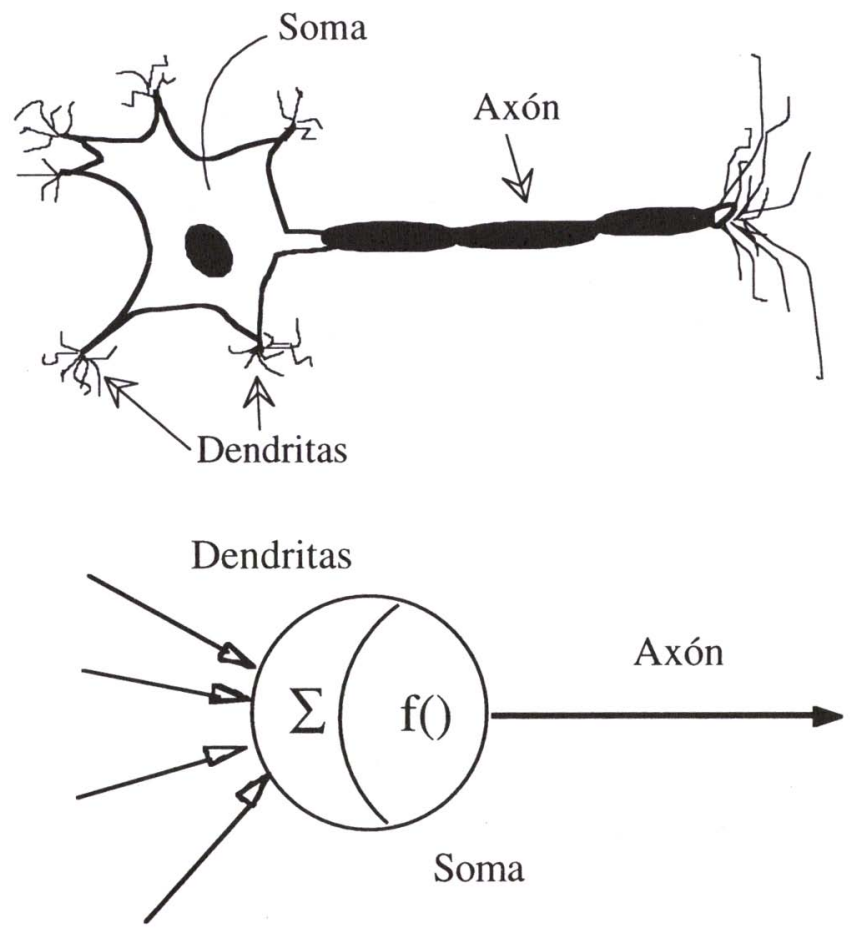

Figura 1. Neurona biológica y esquema de neurona artificial.

Scire. $1: 1$ (en.-jun. 1995). 
de los mejores libros sobre el tema son el de Hecht-Nielsen (1990) y el de Hertz, Krogh y Palmer (1991).

\subsection{Modelo de neurona artificial}

Como sabemos, el punto de vista de los SNA es la computación mediante conjuntos de procesadores sencillos y adaptativos (neuronas), ampliamente conectados y que operan en paralelo. El elemento básico de este esquema es la neurona artificial.

Se denomina procesador elemental o neurona a un dispositivo simple de cálculo (Fig. 1) que, a partir de un vector de entradas $\mathbf{x}(\mathrm{t})$ provenientes del exterior o de otras neuronas, proporciona una única respuesta o salida $y_{i}(t)$. Los elementos esenciales que constituyen la neurona de etiqueta i son los siguientes:

- Un conjunto de entradas $x_{j}(t)$ y de pesos sinápticos $w_{i j}$.

- Una regla de propagación $h_{i}(t)=\mathrm{s}\left(w_{i j}, x_{j}(t)\right)$.

- Una función de activación $y_{i}(t)=f_{i}\left(y_{i}(t-1), h_{i}(t)\right)$, que proporciona su salida $y_{i}(t)$.

Las entradas y salidas de la neurona artificial pueden ser binarias (digitales) o continuas (analógicas), dependiendo del modelo y de la aplicación. Por ejemplo, para tareas de clasificación se emplearían salidas digitales $0,+1$, mientras que en un problema de ajuste funcional de una aplicación multivariable continua, se emplearían salidas continuas dentro de un cierto intervalo.

La regla de propagación, a partir de las entradas de la neurona y de sus pesos proporciona el valor del potencial postsináptico. La regla más habitual es lineal, llevando a cabo la suma de las entradas ponderada con los pesos sinápticos. A esta expresión se suele añadir un parámetro adicional i denominado umbral

$$
h_{i}(t)=\sum_{j} w_{i j} x_{j}-\theta_{i}
$$

En ocasiones se hace uso de sinapsis multiplicativas, denominadas de orden superior o sigma-pi (Rumel86).

La función de activación o de transferencia $f($.) proporciona a partir del potencial postsináptico $h_{i}(t)$ y del propio estado de activación anterior de la neurona $y_{i}(t-1)$, su estado de activación actual $y_{i}(t)$. En la mayor parte de los modelos esta función es monótona creciente y continua, como se observa en muchas de las neuronas biológicas. Las formas funcionales más empleadas en los SNA se muestran en la Figura 2. Las más simples de todas son la función identidad (función lineal) y la escalón, empleada en la neurona original de McCulloch-Pitts. La

Scire. 1 : 1 (en.-jun. 1995). 
función lineal a tramos puede considerarse como una lineal saturada en sus extremos; es de gran sencillez computacional, y más plausible desde un punto de vista biológico. Cuando los algoritmos de aprendizaje requieren que la función de activación sea derivable (como en el de retroprogación) se suelen emplear de tipo
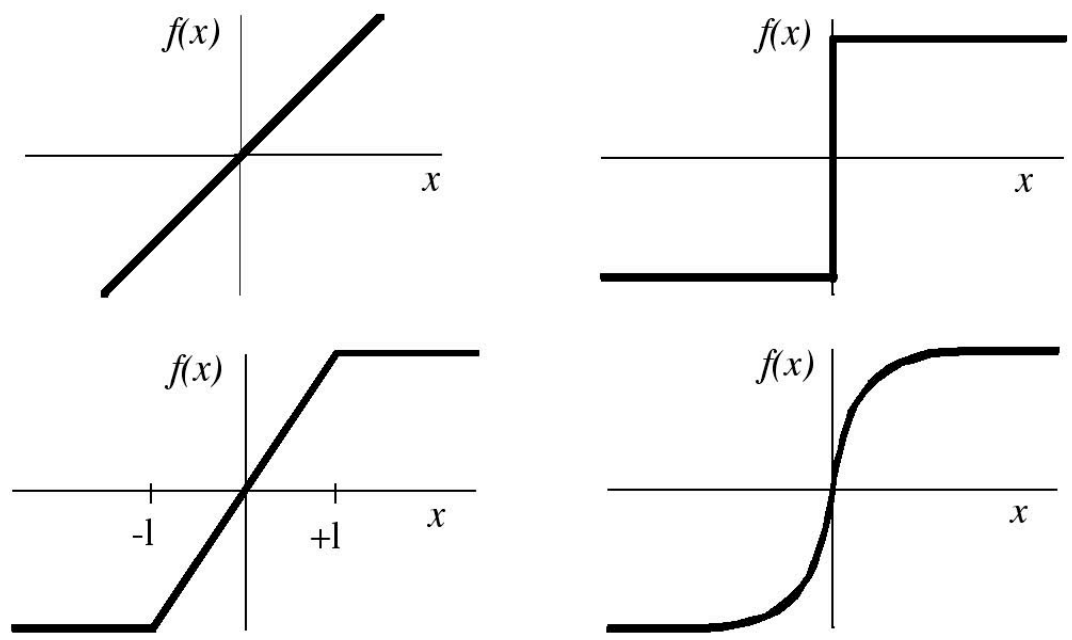

Figura 2. Funciones de activación más habituales:

a) lineal; b) escalón; c) lineal a tramos; d) sigmoidea.

sigmoideo.

Teniendo en cuenta las consideraciones anteriores, el modelo de neurona estándar suele escribirse (Fig. 3) de la siguiente manera:

$$
y_{i}(t)=f_{i}\left(\sum_{j} w_{i j} x_{j}-\theta_{i}\right)
$$

El modelo de neurona considerado hasta el momento es de carácter determinista. Sin embargo, puede introducirse una dinámica estocástica en la activación de la neurona, lo que conduce a que la salida posea carácter probabilístico, como sucede en la máquina de Boltzmann (Rumelhart, 1986). 


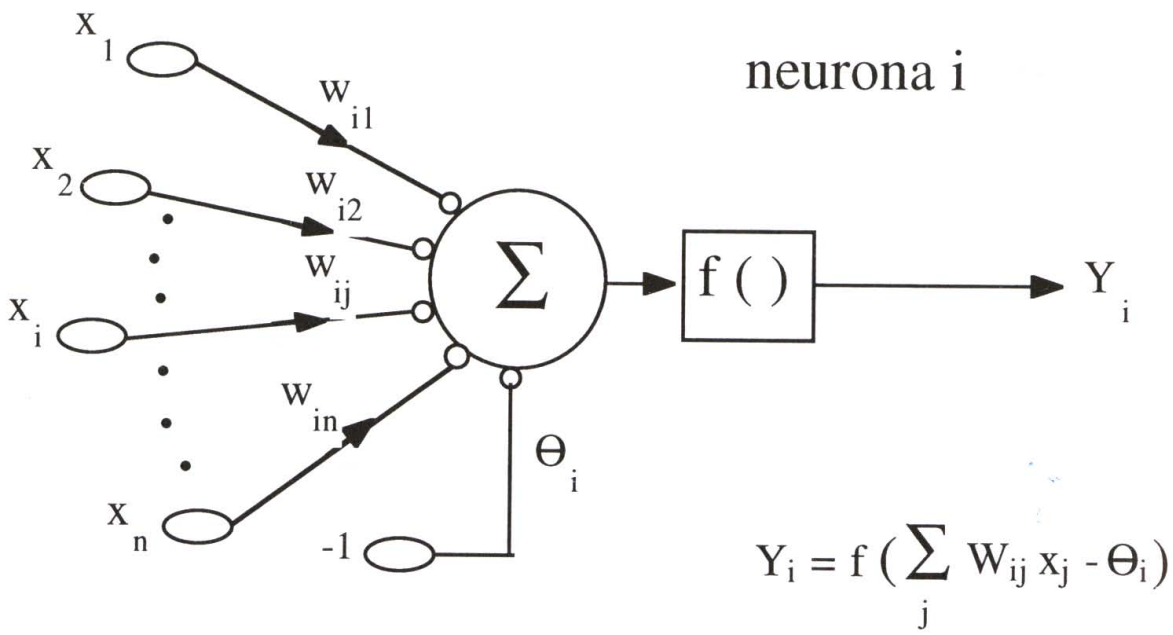

Figura 3. Modelo de neurona estándar.

\subsection{Arquitecturas de redes neuronales}

Se denomina arquitectura a la topología, estructura o patrón de conexionado de una red neuronal. En general, y de modo análogo a lo que sucede en el cerebro, las neuronas se suelen agrupar en unidades estructurales que denominaremos capas. El conjunto de una o más capas conforma la red neuronal.

Se distinguen distintos tipos de capas: la capa de entrada o sensorial está compuesta por neuronas que reciben datos o señales del entorno; la capa de salida es aquella cuyas neuronas proporcionan la respuesta de la red neuronal; las capas ocultas no tienen conexión directa con el entorno, proporcionando a la red neuronal grados de libertad adicionales.

Se pueden distinguir diferentes tipos de arquitecturas neuronales, atendiendo a diferentes aspectos. En relación a su estructura en capas, podemos hablar de redes monocapa, como aquellas compuestas por una única capa de neuronas; y las redes multicapa (layered networks), cuyas neuronas se organizan en varias capas.

Asímismo, atendiendo al flujo de datos en el seno de la red neuronal, podemos hablar de arquitecturas hacia-adelante (feedforward) y arquitecturas recurrentes (feedback). En las redes hacia-adelante, los datos siempre circulan en un

Scire. $1: 1$ (en.-jun. 1995). 


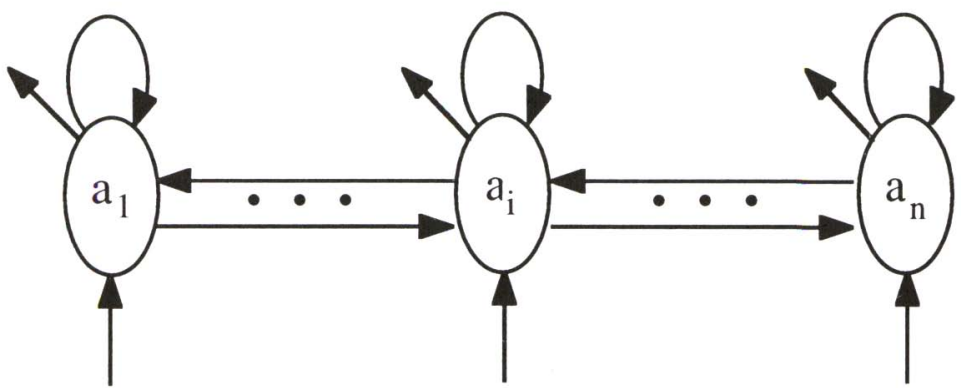

Figura 4. Arquitecturas neuronales.

a) Ejemplo de arquitectura monocapa y realimentada.

b) Ejemplo de arquitectura multicapa y hacia-adelante.

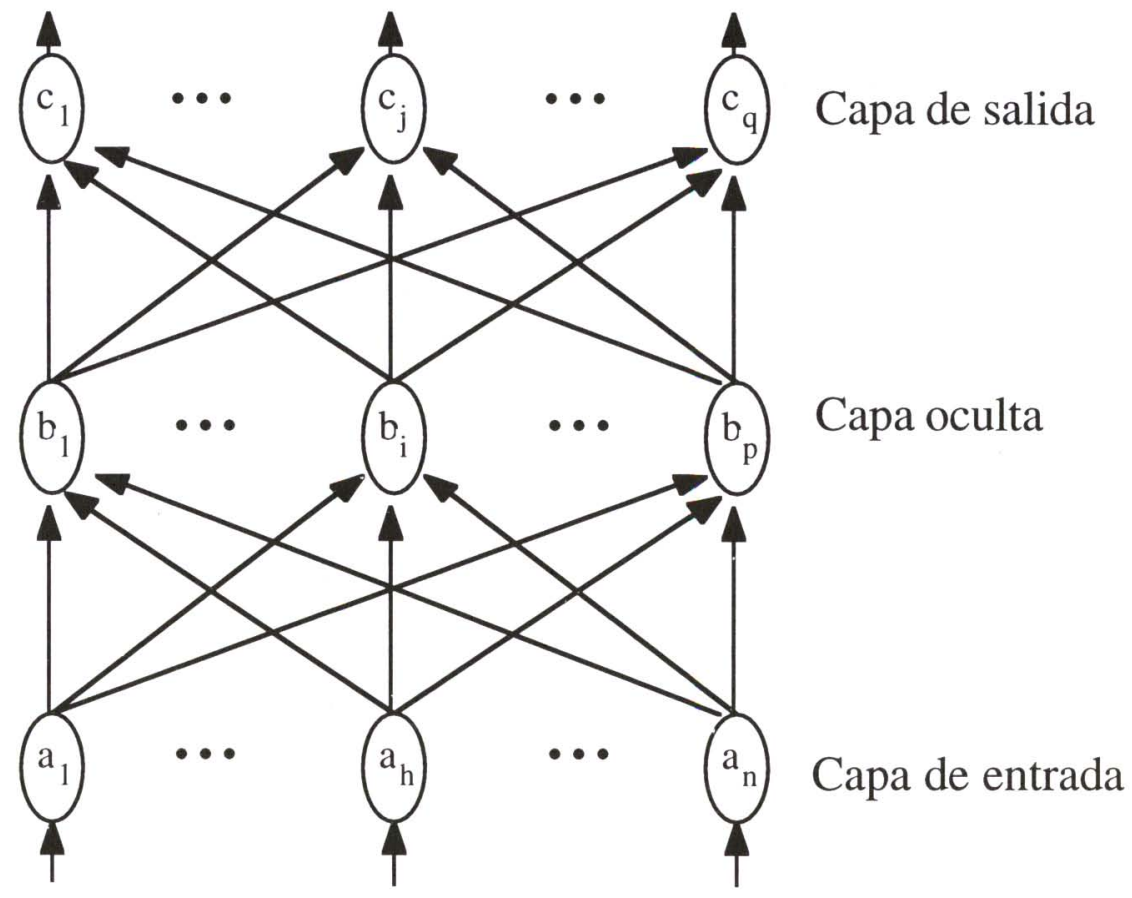


único sentido, desde las neuronas de entrada hacia las de salida. En las arquitecturas recurrentes existe realimentación, de modo que los datos pueden circular también en el sentido salida-entrada, contrario al convencional.

Con frecuencia se interpreta la operación de una red neuronal como la de una memoria asociativa, que ante un patrón de entradas responde con un cierto patrón de salida. Si una red se entrena para que ante la presentación de un patrón A responda con un patrón $B(\neq \mathrm{A})$, se dice que la red es heteroasociativa. Si una red es entrenada para que a un patrón A le asocie el mismo patrón A, se dice que es una red autoasociativa.

\subsection{Modos o dinámicas de operación: recuerdo y aprendizaje}

Definidos los modelos de neurona y su patrón de conexionado, sobre la arquitectura resultante se definen dos dinámicas o modos de operación: el modo recuerdo o de ejecución, y el de aprendizaje o entrenamiento.

\subsubsection{Modo aprendizaje}

Habitualmente, los pesos sinápticos iniciales de la arquitectura se establecen como nulos o aleatorios. Ello quiere decir que la red de partida normalmente no almacena ningún tipo de información útil, para que resulte operativa es necesario entrenarla, fase que constituye el modo aprendizaje de la red, que consistente esencialmente en encontrar un conjunto de pesos sinápticos adecuado que permita a la red realizar el tipo de procesamiento deseado. El aprendizaje se puede llevar a cabo a dos niveles. El más convencional es el del modelado de las sinapsis, que consiste en modificar los pesos sinápticos siguiendo una cierta regla de aprendizaje, que normalmente se obtiene de la minimización de una función de error o coste, que mide el rendimiento actual de la red. Algunos modelos neuronales pueden incluir un segundo nivel en el aprendizaje, el de la creación y/o destrucción de neuronas, en el que se lleva a cabo la modificación de la propia arquitectura de la red. Sea de una manera u otra, en un proceso de aprendizaje la información presente en la capa de entrada modela la estructura de la red, como resultado la red neuronal incorpora en su propia estructura una cierta imagen de su entorno.

Describiremos a continuación los dos tipos básicos de aprendizaje, el supervisado y el no supervisado:

Aprendizaje supervisado. En este tipo se presenta a la red un conjunto de patrones, junto con la salida deseada u objetivo. La red iterativamente ajusta sus pesos hasta que su salida tiende a ser la deseada, utilizando para ello información detallada del error que en cada paso comete. De este modo, el SNA es capaz de estimar relaciones entrada/salida sin necesidad de proponer una cierta forma funcional de partida.

Scire. 1 : 1 (en.-jun. 1995). 
Aprendizaje no supervisado o autoorganizado. En este caso se presentan a la red multitud de patrones, pero sin adjuntar la respuesta deseada. El sistema, por medio de la regla de aprendizaje definida, debe reconocer en ellos regularidades, extraer rasgos, estimar densidades de probabilidad o agruparlos según su similitud.

El tipo de aprendizaje más común es el supervisado. Suele ser además computacionalmente más complejo que el autoorganizado, pero también más exactos sus resultados. No obstante, ambas clases tienen sus propias aplicaciones.

Además de las formas básicas comentadas, podemos distinguir dos tipos de aprendizaje adicionales. Uno es el aprendizaje híbrido, que consiste en la coexistencia en una misma red neuronal de aprendizaje supervisado y autoorganizado (normalmente en distintas capas). Otro tipo es el aprendizaje reforzado (reinforcement learning), que se sitúa a medio camino entre el supervisado y el autoorganizado, y en el cual se proporciona una única señal de error global.

Para comprender cómo opera el entrenamiento en una red neuronal, pondremos un ejemplo de aprendizaje supervisado. Supongamos que debemos desarrollar un sistema de reconocimiento de números escritos a mano. De partida se propondría una cierta arquitectura neuronal, cuyas entradas serían los pixeles que componen la imagen de la cifra a interpretar. La red podría tener 10 neuronas de salida, una por cada dígito (del 0 al 9), de modo que la activación de una de estas neuronas indicaría cuál es la cifra que ha sido presentada. Definida la arquitectura, se procede a su entrenamiento. Este se inicia con la recolección de un buen número de ejemplos, consistentes en diferentes cifras manuscritas, que constituirían el conjunto de aprendizaje. Una parte se mantendrá al margen del entrenamiento para poder realizar al final un test objetivo del sistema desarrollado. Los patrones que constituyen el conjunto de aprendizaje se presentan una y otra vez a la red, y sus pesos se van modificando. Por ejemplo, ante un carácter ' 0 ' la red respondería al principio con un vector de salidas incorrecto, que compararíamos con el valor objetivo (la primera neurona activada y todas las demás desactivadas), calculando el error que está cometiendo la red en ese instante para el patrón presentado. A continuación se procede a la modificación de los pesos con la intención de reducir el error en cierta medida. Posteriormente presentaríamos un ' 1 ', un '7', un '2', etc., comparando cada vez la salida que proporciona la red con la deseada, y modificando los pesos para reducir el error. Con un número suficiente de iteraciones, la red aprendería a asociar todas las cifras del '0' al '9' con su salida correcta. Por último, con los patrones del conjunto de test mediríamos objetivamente la eficacia real del sistema, ante patrones nunca antes presentados.

Como se apunta en el párrafo anterior, en el aprendizaje, siempre hay que distinguir entre el nivel de error al que se llega al final del entrenamiento para el con-

Scire. 1 : 1 (en.-jun. 1995). 
junto de datos de aprendizaje, y el error que la red ya entrenada comete ante patrones nunca antes presentados. Es ésta última la denominada capacidad de generalización de la red, lo que constituye uno de los asuntos fundamentales en el entrenamiento. El objetivo es siempre alcanzar la mejor generalización pues indicará que el sistema desarrollado es capaz de tratar correctamente con patrones nuevos, y que es suficientemente general. Existen diferentes maneras de garantizar una buena generalización, entre las cuales destaca la técnica de validación cruzada (Hecht-Nielsen, 1990).

\subsubsection{Modo recuerdo o de ejecución}

Una vez el sistema ha sido entrenado, en la mayor parte de los modelos el aprendizaje se desconecta, los pesos y la estructura permanecen fijos, y la red neuronal queda dispuesta para procesar información, proporcionando una respuesta ante un patrón o vector de entrada. Es éste el modo recuerdo (recall) o de ejecución.

En las redes hacia-adelante, dado un patrón de entrada, las neuronas responden y proporcionan directamente la salida. $\mathrm{Al}$ no haber bucles de realimentación no existe ningún problema en relación a su estabilidad. Ahora bien, en una red con realimentaciones, la estabilidad de la red no queda garantizada. Las redes realimentadas son sistemas dinámicos no lineales, que ante una entrada solamente en determinadas condiciones responderán de una manera estable, convergiendo a un punto fijo de la red o estado estable. Existen una serie de teoremas generales, como los de Cohen-Grossberg y Cohen-Grossberg-Kosko (Kosko, 1992), que aseguran la estabilidad de la respuesta en una amplia clase de redes neuronales, basándose para ello en el empleo del método de Lyapunov.

\subsection{Computabilidad neuronal}

Es sabido que los ordenadores digitales convencionales son máquinas universales de Turing, por lo que pueden llevar a cabo cualquier tipo de cómputo. Los SNA aparecen como alternativas y/o complemento a los sistemas de cálculo convencionales, por lo que nos podemos preguntar qué tipo de cómputo pueden llevar a cabo estos sistemas, en particular si también se trata de máquinas universales.

En (Abu-Mostafa, 1986) se trata extensamente este asunto, demostrándose que, al igual que los ordenadores digitales, las redes neuronales pueden resolver cualquier problema, es decir, se trata también de dispositivos universales de computación. Estudiando diversos aspectos relacionados con la complejidad computacional, el autor llega a la conclusión de que aunque ambos esquemas de cómputo son universales, cada uno está mejor indicado para la ejecución de una cierta clase de tareas. Así, un ordenador digital se mostrará más eficaz en la resolución

Scire. 1 : 1 (en.-jun. 1995). 
de problemas que precisen de algoritmos de longitud corta. Sin embargo, en aquellos en los que se deba almacenar una gran base de datos y se requieran algoritmos de gran tamaño, una red neuronal proporciona una solución más eficiente. Es decir, un sistema convencional ejecutará eficazmente tareas del tipo de razonamiento lógico o aritméticos, mientras que un SNA proporcionará una mejor solución a problemas del tipo de los de reconocimientos de formas, en los que existen numerosos casos particulares que deben almacenarse. Un caso típico es el del reconocimiento de imágenes en ambiente natural. Por ejemplo, para determinar a qué clase pertenece el árbol que aparece ante una cámara de vídeo, se debería tener almacenada en memoria una gran base de datos compuesta por diferentes clases de árboles y ejemplos de cada clase, para poder comparar.

Un área de intenso trabajo en la actualidad es la de comparar las técnicas neuronales y las estadísticas, pues al fin y al cabo ambas tratan de resolver problemas similares mediante métodos en ocasiones sospechosamente parecidos. En este sentido, afortunadamente se está empezando a desterrar la falsa idea de considerar las redes neuronales como dispositivos mágicos capaces de resolver cualquier problema de una manera maravillosa, y a ello está contribuyendo poderosamente el estudio comparativo entre estadística y SNA. El asunto es de sumo interés, pero se escapa a los propósitos y extensión del presente trabajo. Una excelente visión del tema se muestra en (NATO, 1994), que próximamente será publicado por una editorial internacional.

\section{Modelos de redes neuronales artificiales}

Existen multitud de modelos de SNA. De ellos, unos cincuenta son medianamente conocidos y una quincena bastante populares. Como es fácil comprender, se precisa de una clasificación de los modelos para establecer algo de orden en su estudio. En la Fig. 5 se muestra una posible clasificación atendiendo al tipo de aprendizaje (supervisado, no supervisado, híbrido y reforzado) y a la topología de la red (redes realimentadas y redes hacia-adelante). El libro de Wasserman (1989) constituye una correcta y sencilla introducción a estos modelos.

La clase de modelos más importante la constituyen las redes hacia-adelante y de aprendizaje supervisado. A ella pertenecen el perceptrón, la adalina y el perceptrón multicapa. El perceptrón multicapa es el modelo neuronal más conocido y empleado en la práctica. Suele emplearse junto al aprendizaje de retropropagación (backpropagation), por lo que habitualmente se denomina simplemente red de retropropagación o BP (Rumelhart, 86). Su importancia se debe a su potencia y generalidad, pues se ha demostrado que constituye un aproximador universal de funciones (Hornik, 1989), lo que hace de él uno de los modelos más útiles en la práctica. La operación de este sistema se relaciona con la regresión no lineal.

Scire. $1: 1$ (en.-jun. 1995). 


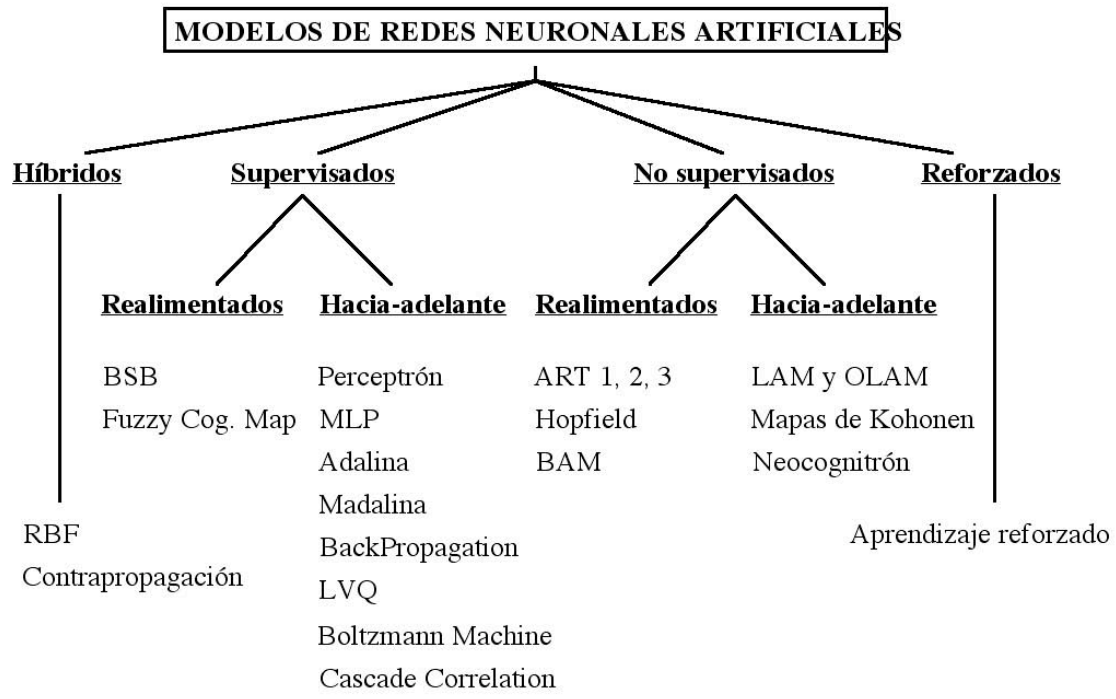

Fig. 5. Clasificación de los modelos de redes neuronales artificiales.

De entre todos los modelos no supervisados se pueden distinguir el ART, neocognitrón y las redes PCA (Wasserman, 1989). No obstante, los mapas autoorganizados o SOFM (Self-Organizing Feature Maps) (Kohonen, 1990) son posiblemente el más popular de todos. Este sistema neuronal reproduce el tipo de mapas sensoriales existentes en el córtex cerebral. Los SOFM, en esencia, realizan la proyección no lineal de un espacio multidimensional de entrada sobre las dos dimensiones del mapa, preservando los rasgos originales más importantes. Por este motivo se emplea con frecuencia en la visualización de complejos conjuntos de datos.

Uno de los modelos realimentados más conocidos y estudiados es el modelo de Hopfield (Hopfield, 1982). Consiste en una red completamente interconectada, de tipo autoasociativo y recurrente, que en su versión discreta se utiliza como memoria asociativa, y en su versión continua se aplica a la resolución de problemas de optimización. Se ha establecido su equivalencia con los modelos de spinglass procedentes de la mecánica estadística, por lo que sobre él se está realizando un gran trabajo teórico (Domany, 1991).

Finalmente, uno de los modelos híbridos más destacables, es el de funciones de base radial o $R B F$ (Radial Basis Functions). Se estructura en tres capas, con la 
intermedia de tipo autoorganizado de nodos gaussianos, y la segunda supervisada. Un excelente introducción al RBF puede encontrarse en (Hush, 1993).

Estos modelos, junto a muchos otros, se encuentran ampliamente explicados en (Hertz, 1991; Wasserman, 1989; Hecht-Nielsen, 1991; Hush, 1993), referencias a las que remitimos al lector interesado en ampliar los detalles.

\section{Realización de redes neuronales artificiales}

Establecidos los fundamentos de los SNA, veremos a continuación las diferentes maneras de construirlos en la práctica. El modo más habitual de trabajo con SNA consiste en su simulación mediante programas de ordenador escritos en lenguajes de alto nivel como $\mathrm{C}$ o Pascal, que se ejecutan en ordenadores convencionales, como pueda ser un PC compatible o una estación de trabajo. Aunque de esta manera se pierde su capacidad de cálculo en paralelo, las prestaciones que ofrecen los ordenadores actuales resultan suficientes para resolver numerosos problemas prácticos, al permitir simular redes de tamaño considerable a una velocidad razonable. Esta constituye la manera más barata y directa. En este caso, la actividad de cada neurona se suele representar mediante una variable del programa. Como las neuronas se agrupan en capas, las actividades de las neuronas de una capa se suelen reunir en vectores. Lo mismo sucede con las salidas.

float x[N_INPUTS], y[N_OUTPUTS]; /* Entradas $\mathbf{x}$ y salidas $\mathbf{y}$ de una capa $* /$

Cada neurona posee un vector de pesos sinápticos, por lo tanto, los pesos correspondientes a las neuronas de una capa se agrupan en una matriz $\mathrm{W}$ que los almacena

\section{float w[N_OUTPUTS][N_INPUTS]; /* Matriz de pesos sinápticos */}

De este modo, las salidas y de las neuronas de la capa en cuestión se calcularán a partir del producto de sus entradas x por la matriz de pesos $\mathrm{W}$, resultado al que se aplica la función de transferencia f() (ecuación 2, en la que hemos eliminado el umbral por claridad)

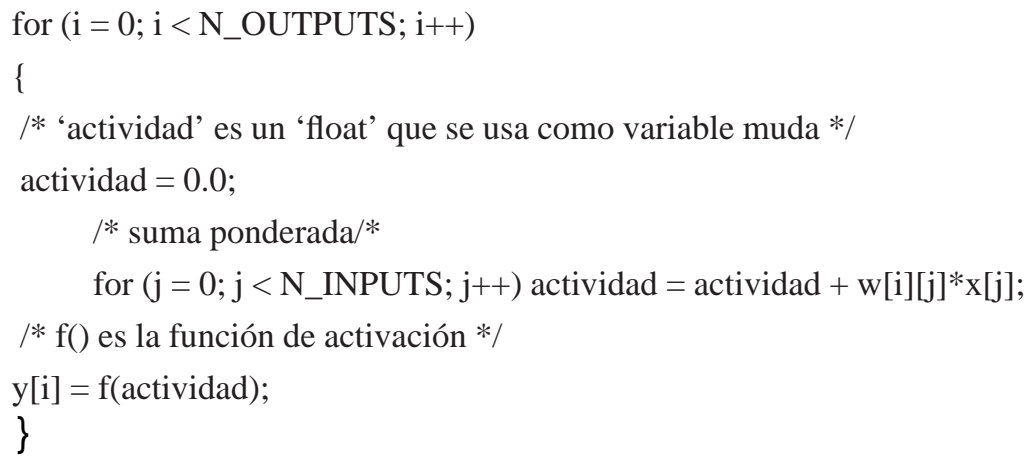

Scire. $1: 1$ (en.-jun. 1995). 

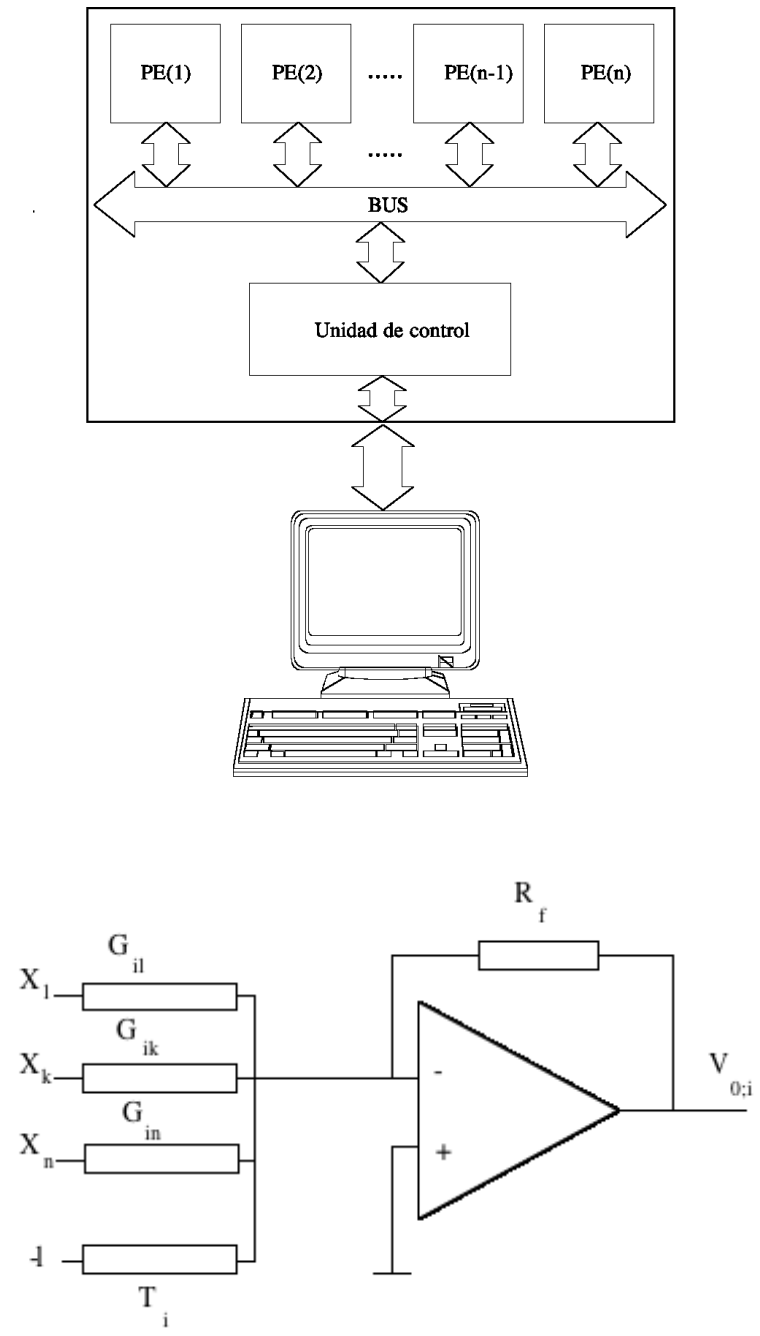

Fig. 6. Dos ejemplos de realización hardware de redes neuronales:

a) Coprocesador neuronal digital. b) Circuito analógico sencillo, basado en un amplificador operacional, que representa una neurona.

En general no es necesario que cada diseñador confeccione sus propios simu- 
ladores, pues existen sistemas de desarrollo comerciales para SNA, consistentes en un software de simulación que permite el trabajo con multitud de modelos neuronales. En Hammerstrom (1993a) se citan unos cuantos ejemplos.

En el resto de las maneras de realizar SNA se trata de aprovechar en mayor o menor medida su estructura de cálculo paralelo. Así, un paso adelante en este sentido consiste en simular la red sobre un computador de propósito general con capacidad de cálculo paralelo (redes de transputers, máquinas vectoriales, máquinas paralelas, ...).

Una orientación diferente consiste en llevar a cabo la emulación hardware del SNA. Con el término emulación nos referimos al empleo de sistemas de cálculo expresamente diseñados para realizar SNA basados en, bien microprocesadores más o menos convencionales (RISC, DSP, ...), bien en procesadores especialmente diseñados para realizar las operaciones que más frecuentemente emplean las redes neuronales. Estas estructuras se suelen denominar aceleradores o neurocomputadores de propósito general. Algunos sistemas de desarrollo de redes neuronales, además de un software de simulación, incluyen dispositivos de este tipo, en forma de tarjetas adaptables al bus de un computador convencional, lo que permite acelerar notablemente las simulaciones de SNA. Un ejemplo es la tarjeta Balboa 860, de la Hecht-Nielsen Inc., basada en el microprocesador RISC i860.

El aprovechamiento a fondo de la capacidad de cálculo masivamente paralelo de los SNA pasa por la realización hardware de la estructura de la red neuronal, en forma de circuitos específicos que reflejan con cierta fidelidad la arquitectura de la red. Estas estructuras se denominan neurocomputadores de propósito específico. La tecnología más habitualmente empleada es la microelectrónica VLSI (Cabestany, 1993) (Very Large Scale Integration, o integración a muy alta escala). Los circuitos integrados así construidos se suelen denominar chips neuronales. No obstante, también se están desarrollando redes optoelectrónicas e, incluso, redes basadas en procesos biotecnológicos, aunque se trata más de promesas que de realidades. La realización hardware de la red neuronal es la manera de poder aplicar estos sistemas a la resolución de problemas que involucran un gran número de datos y precisan respuestas en tiempo real (por ejemplo, un sistema de detección e interceptación de misiles enemigos (Lupo, 1989).

Hay que señalar que una de las causas más importantes del renacimiento de las redes neuronales fue precisamente el desarrollo de la integración VLSI, que influyó decisivamente en la explosión de interés en los SNA a causa de dos circunstancias. Por una parte, la integración microelectrónica a muy alta escala permitió el desarrollo de computadores potentes y baratos, lo que facilitó la simulación de modelos de redes neuronales artificiales de un alto nivel de complejidad.

Scire. 1 : 1 (en.-jun. 1995). 
De este modo, los SNA pudieron aplicarse a mediados de los años ochenta a numerosos problemas prácticos concretos, gracias a lo cual se comprobó su gran potencial. Por otra parte, la integración VLSI posibilitó la realización hardware de los SNA, abriendo un nuevo mundo de posibilidades a estos sistemas, como dispositivos de cálculo paralelo aplicables a problemas computacionalmente costosos, como puedan ser los de visión o reconocimiento de patrones.

La realización electrónica de redes neuronales es un campo tremendamente activo, que está siendo abordado no solo por grupos universitarios de todo el mundo, sino también por las grandes empresas de los sectores de la electrónica e informática, como Intel, Siemens, Philips, Hitachi, AT\&T y otras (Cabestany, 1993; Hammerstrom, 1993). Cada empresa mantiene su propio programa de I+D en relación al tema. Fruto de ello es la aparición de los primeros productos comerciales de importancia, como el procesador neuronal ETANN de Intel o el neurocomputador CNAPS de Adaptive Solutions. Algunas de las realizaciones más espectaculares se deben al grupo del norteamericano Carver Mead (Mead, 1989), con el desarrollo de sistemas neuronales electrónicos que imitan la estructura neuronal de órganos sensoriales como la retina (Mahowald, 1991) o la cóclea, que ofrecen unos resultados sorprendentemente similares a los sistemas biológicos que emulan (aunque del orden de un millón de veces más rápidos).

De todos modos, la realización hardware de redes neuronales constituye un área de trabajo muy joven, en el que la tecnología se explota al límite de sus actuales posibilidades. Por ejemplo, en un sistema tan complejo como la retina electrónica de Mead se pueden integrar hasta unos pocos miles de neuronas, cuando la retina biológica que trata de emular está compuesta por millones, lo que nos da una idea de lo lejos que nos encontramos todavía de la construcción de un cerebro electrónico.

\section{Aplicaciones.}

Son diversas las características de las redes neuronales que pueden resultar interesantes para la resolución de problemas. Una de ellas es su capacidad de aprendizaje, que les permite aprender a ejecutar una tarea por medio de ejemplos, a partir de los cuales puede generalizar. Si a ello añadimos su no linealidad, las redes neuronales se configuran como potentes estimadores de funciones complejas. Otras características de interés son su tolerancia a la imprecisión e incertidumbre presentes en el entorno, y su capacidad de cálculo paralelo, que resulta fundamental en numerosos problemas estratégicos en los que se deben tomar importantes decisiones en fracciones de segundo (Lupo, 1989).

Como cualidad negativa se suele señalar que los SNA presentan dificultades a la hora de explicar su operación. Los importantes recursos de cálculo que se 
precisan para el trabajo con SNA, especialmente en la fase de aprendizaje, se suele señalar como otro de sus puntos negros. Por último, al tratarse de un campo joven y en evolución, los resultados teóricos se van asentando poco a poco, quedando todavía mucho trabajo por hacer.

En la actualidad, las aplicaciones prácticas de los SNA son muy numerosas. Los dos artículos de Hammerstrom (1993a, 1993b) representan un estupendo repaso a sus numerosos campos de aplicación. Los más habituales son los relacionados con la clasificación, la estimación funcional y la optimización. En general, el del reconocimiento de patrones suele considerarse como un denominador común. Areas de aplicación son las siguientes: reconocimiento del habla, reconocimiento de caracteres manuscritos, visión, robótica, control, predicción, economía, defensa, bioingeniería, etc. Asímismo, las redes neuronales se están aplicando también a la extracción de reglas para incorporar en sistemas difusos, así como a la confección de los denominados sistemas expertos conexionistas, donde se conjugan redes neuronales y las técnicas de los sistemas expertos (Gallant, 1993).

Así como las aplicaciones prácticas resultaban hace unos años anecdóticas o estaban en fase experimental, en la actualidad muchas compañías importantes las aplican de un modo rutinario a numerosos problemas.

A modo de ejemplo, Sharp en Japón ha desarrollado un sistema de reconocimiento de caracteres para el alfabeto Kanji (japonés) utilizando una red neuronal jerárquica basada en el modelo neuronal LVQ (Kohonen, 1990). Por su parte, Synaptics, empresa situada en el Silicon Valley, ha desarrollado un chip neuronal para el reconocimiento de direcciones escritas en los sobres de las cartas (Hammerstrom, 1993a).

Un área de intenso trabajo es el del tratamiento de la información económica. En este campo, uno de los grupos punteros es el de A.N. Refenes, de la London Business School, quienes han desarrollado varios sistemas de predicción de parámetros económicos, especialmente pensados para inversión, que superan el rendimiento de los métodos convencionales (Refenes, 1994). En esta misma área de trabajo, hemos desarrollado un sistema de predicción de quiebras combinando mapas autoorganizados (Martín y Serrano, 1993), perceptrones y técnicas estadísticas convencionales.

Fujitsu, Kawasaki y Nippon Steel Corp. han desarrollado sistemas neuronales que se emplean en el control procesos industriales, como en plantas de producción de acero. Citröen emplea redes neuronales en la determinación de la calidad del material utilizado en la confección de los asientos de los vehículos. Mercedes Benz y Ford estudian el empleo de chips neuronales para ahorrar combustible o predecir fallos (Hammerstrom, 1993a). Por último, la nueva versión

Scire. $1: 1$ (en.-jun. 1995). 
del avión de combate F-15 incluye un sistema neuronal para ayudar al piloto en su gobierno en caso de que sea alcanzado por el fuego enemigo (Freedman, 1994), aprovechando que una red neuronal electrónica puede aprender a desenvolverse en las nuevas circunstancias que influyen en el pilotaje del avión miles de veces más rápidamente que el ser humano.

\section{Conclusiones}

En este trabajo hemos realizado una introducción a las redes neuronales artificiales, en sus diferentes aspectos: fundamentos, realización hardware y software y aplicaciones. Esta es un área de trabajo multidisciplinar y de dinámico desarrollo, que empieza a configurarse como de gran interés para la resolución de problemas prácticos de mundo real.

En un próximo trabajo, mostraremos ejemplos de aplicación práctica haciendo uso de datos pertenecientes a problemas reales, donde se mostrará cómo operan algunos de los modelos más populares y cómo se puede llevar a cabo el desarrollo de una aplicación con redes neuronales.

\section{Notas}

(1) En (Marks, 1993) se realiza un estudio comparativo sobre los artículos y patentes desarrollados durante los últimos años en inteligencia computacional (redes neuronales y sistemas difusos, especialmente) e IA. En cuanto a artículos, ha disminuido el número de los publicados tanto en IA en general, como en sistemas expertos en particular, aumentando espectacularmente el trabajo en redes neuronales. En cuanto a patentes, el número sigue creciendo en todas las disciplinas citadas, pero el ritmo es mucho mayor en redes neuronales.

(2) Los computadores digitales convencionales son de este tipo, cuya característica fundamental es que ejecutan secuencialmente las instrucciones de programa almacenadas en memoria.

(3) Microprocesadores especializados en el procesamiento digital de señal.

\section{Referencias.}

Abu-Mostafa, Y. S. (1986). Neural networks for computing? // Denker, J. S. (ed.). Neural networks for computing: AIP proceedings. 151 (1986) 1-7.

Cabestany, J. ; Castillo, F. ; Moreno, M. (1983). Redes Neuronales : realizaciones en circuitos integrados. // Mundo Electrónico. 239 (1993) 24-31.

Conrad, M. (1992). Molecular computing paradigms. // IEEE Computer. 6-9 (Dec. 1992).

Domany, E. ; van Hemmen, J. L. ; Schulten, K. (eds.) (1991). Models of Neural Networks. Springer-Verlag, 1991.

Freedman, D. H. (1994). A romance blossoms between gray matter and silicon. // Science. 265 (Ag. 1994) 889-890.

Gallant, S.I. (1993). Neural Network Learning and Expert Systems. MIT Press, 1993.

Scire. $1: 1$ (en.-jun. 1995). 
Hammerstrom, D. (1993a). Neural Networks at work. // IEEE Spectrum. (June 1993) 2632.

Hammerstrom, D. (1993b) Working with Neural Networks. // IEEE Spectrum. (July 1993) 46-53.

Hecht-Nielsen, R. (1990). Neurocomputing. Addison Wesley, 1990.

Hertz, J. ; Krogh, A. ; Palmer, R. (1991). Introduction to the theory of Neural Computation. Addison-Wesley, 1991.

Hopfield, J. J. (1982). Neural networks and physical systems with emergent collective computational abilities. // Proc. of the Nat. Acad. of Sci. 79 (1982) 254-58.

Hornik, K. ; Stichcombe, M. ; White, H. (1989). Multilayer feedforward networks are universal approximators. // Neural Networks. 2 (1989) 359-366.

Hush, D. R. ; Horne, B. G. (1993). Progress in supervised neural networks. What's new since Lippmann?. // IEEE Signal Processing Magazine. (Jan. 1993) 8-38.

Kohonen, T. (1990). The Self-Organizing Map. // Proc. of the IEEE. 78 : 9 (1990) 146480.

Kosko, B. (1992). Neural Networks and Fuzzy Systems. Prentice-Hall Int., 1992.

Lippmann R. P. (1987). An introduction to computing with neural nets. // IEEE ASSP Magazine. (Ap. 1987) 4-22, 1987.

Lupo, J. C. (1989). Defense applications of neural networks. // IEEE Communications Magazine. (Nov. 1989) 82-88.

Mahowald, M. ; Mead, C. (1991). La retina de silicio. // Investigación y Ciencia. (Jul. 1991) 42-49.

Marijuan, P. C. (1992). La acumulación social del conocimiento: una perspectiva interdisciplinar. // Jornadas de Organización del Conocimiento, Zaragoza, nov. 1992.

Marks II, R. J. (1993). Intelligence: Computational versus artificial. // IEEE Trans. on Neural Networks. 4 : 5 (1993) 737-739.

Martín del Brío, B. ; Serrano Cinca, C. (1993). Self-organizing neural networks for analysis and representation of data : some financial cases. // Neural Computing and Applications, 1. London, 1993. 193-206.

Mead, C. (1989). Analog VLSI and Neural Systems. Addison-Wesley, 1989.

NATO Advanced Study Institute (1994). NATO ASI From Statistics to Neural Networks. Les Arcs (France), June, 1994.

Refenes, A.N. ; Zapranis, A. ; Francis, G. (1994). Stock performance modeling using neural networks : a comparative study with regression models. // Neural Networks. $7: 2$ (1994) 375-388.

Rumelhart, D.E. ; McClelland, J. L. (eds.) (1986). Parallel distributed processing. Vol 1: Foundations. Vol 2: Psychological and biological models. MIT Press, 1986.

Wasserman, P. D. (1989). Neural Computing : Theory and Practice. Van Nostrand Reinhold, 1989.

Scire. 1 : 1 (en.-jun. 1995). 